\title{
Metaanálisis en red
}

\section{Network Meta-analysis}

\author{
Christian Fau*, Solange Nabzo y Veronica Nasabun \\ Centro Adherente Cochrane Fundación Oftalmológica 2020, Iberoamerican Cochrane Network, Santiago, Chile
}

\begin{abstract}
Resumen
El metaanálisis en red (también llamado comparaciones mixtas de tratamientos) es una poderosa técnica estadística que combina diferentes estudios para realizar el análisis de tratamientos múltiples o estimar un efecto indirecto en ausencia de una comparación directa. Estos estudios se realizan mediante el desarrollo de una red de análisis, lo que permite calcular los efectos relativos de todos los tratamientos o intervenciones incluidos en la red simultáneamente, utilizando técnicas que estiman los análisis directos e indirectos de la evidencia. Las comparaciones indirectas son comparaciones de diferentes tratamientos utilizando datos de distintos estudios mediante un comparador en común, ya sea porque no existen estos estudios o son de baja calidad o bien porque se desea comparar numerosas alternativas. En el metaanálisis en red, la comparación mixta del tratamiento está basada en una red de bucles cerrados. Estos proveen mucha más información y está menos sesgada que la de los bucles abiertos. Actualmente, en los bucles o ciclos cerrados se utilizan varios métodos estadísticos para su análisis, pero en cada estudio se utiliza un enfoque estadístico único. Los más frecuentes hasta la fecha son los métodos bayesianos. Por lo tanto, es más importante el análisis del proceso de investigación y la red creada que la obtención de una medida única ponderada final. El objetivo de esta revisión narrativa es describir los conceptos fundamentales de los metaanálisis en red, su utilidad, las consideraciones metodológicas, los fundamentos del análisis, la conformación de la red y sus principales limitaciones.
\end{abstract}

Palabras clave: Metaanálisis en red. Comparaciones indirectas. Comparación de tratamientos mixtos. Comparación de tratamientos múltiples. Metaanálisis.

\section{Abstract}

The network meta-analysis (also called mixed comparisons of treatments) is a powerful statistical technique that combines different studies to realize the analysis of multiple treatments or to estimate an indirect effect in the absence of a direct comparison. These studies are carried out by the development of a network of analysis, allowing to calculate the relative effects of all the treatments or interventions included in the network simultaneously and using techniques that estimate the direct and indirect analysis of the evidence. Inductions are comparisons of different treatments using data from different studies and using a comparator in common, either because these studies are not available or are of poor quality or if one wishes to compare numerous alternatives. In the network meta-analysis the mixed treatment comparison is based on a closed-loop network, which provides much more information and is less biased than open loops. Currently in closed loops or

Correspondencia:

${ }^{*}$ Christian Fau

Fundación Oftalmológica 2020

Avd. Presidente Riesgo 5157, Dep. $212 \quad$ Fecha de recepción: 19-09-2017

Disponible en internet: 14-05-2018 Las Condes, Santiago, Chile

Fecha de aceptación: 01-03-2018 Rev Mex Oftalmol. 2018;92(3):153-159 E-mail: cfau@fundacion2020.org DOI: 10.24875/RMO.M18000032 www.rmo.com.mx 0187-4519/@ 2018 Sociedad Mexicana de Oftalmología. Publicado por Permanyer México. Este es un artículo Open Access bajo la licencia CC BY-NC-ND (http://creativecommons.org/licenses/by-nc-nd/4.0/). 
cycles, several statistical methods are used for its analysis, but in each study a unique statistical approach is used. The most frequent to date are the Bayesian methods, therefore is more important the analysis of the process of research and network created that obtaining a single weighted final measure. The objective of this narrative review is to describe the fundamental concepts of the network meta-analysis, utility, methodological considerations, the fundamentals of the analysis, the conformation of the network and its main limitations.

Key words: Network meta-analysis. Indirect comparisons. Mixed treatment comparisons. Multiple treatment comparison. Meta-analysis.

\section{Introducción}

Cualquier persona que se enfrente a la tarea de tomar una decisión en salud que afecta a millones de personas o que revista riesgos importantes para la salud desea que su decisión sea lo más objetiva posible y basada en la mejor evidencia disponible. En la práctica, esto normalmente significa encontrar y combinar los resultados de diferentes investigaciones y buscar las mejores alternativas de intervención sanitaria. Este proceso de decisión es dinámico en el tiempo, ya que el número de intervenciones sanitarias disponibles para resolver un problema de salud aumenta con los años y, en muchas áreas de la medicina, muy aceleradamente. Esto obliga a tener una estructura de análisis que incorpore todas las alternativas existentes frente a un tema y en la que, además, se vayan incorporando las nuevas alternativas cada año, lo que permite tener siempre en claro las diferencias entre todas las alternativas. Esto es una red de análisis que crece en el tiempo. En las últimas décadas, producto de lo antes expuesto, se han desarrollado los metaanálisis en red, que son una técnica estadística que permite combinar distintos estudios con iguales o diferentes conjuntos de tratamientos para crear un análisis global. Esto se realiza mediante el desarrollo de una red de análisis que permita calcular los efectos relativos de todos los tratamientos incluidos en la red. Estos efectos se estiman utilizando tanto los análisis directos como los indirectos entre tratamientos ${ }^{1-5}$.

En las últimas décadas se ha aceptado en general que los ensayos clínicos controlados y bien diseñados proporcionan la mejor evidencia para la toma de decisiones, la evidencia más rigurosa y válida en relación con los efectos relativos entre diferentes intervenciones. En la búsqueda de la evidencia disponible para la toma de una decisión es frecuente encontrar más de un ensayo clínico controlado: al aumentar el nivel de información puede aumentar el nivel de precisión en la toma de la decisión. Los estudios pueden ser agrupados mediante la técnica de una revisión sistemática, que permite, mediante un protocolo riguroso, mejorar la selección de estudios según su calidad metodológica. Cuando el diseño de los estudios lo permite, se pueden combinar los resultados disponibles de varios de ellos de forma cuantitativa, mediante un metaanálisis de los datos, y así obtener un estimador más preciso al aumentar el número de pacientes al unir matemáticamente los estudios. La comparación frente a frente (intervención vs. placebo, etc.) de las alternativas de tratamiento en los ensayos clínicos controlados, también llamada análisis directo, es la forma más fiable de comparación entre los tratamientos ${ }^{1-5}$, pero a medida que el número de tratamientos disponibles aumenta, el número de posibles comparaciones entre ellos aumenta exponencialmente. Son pocos los ensayos clínicos que comparan cuatro o más intervenciones diferentes en un mismo estudio, ya que esto encarece mucho su realización. Lo más frecuente es que existan ensayos clínicos controlados solo para una pequeña fracción de las posibles intervenciones a comparar (generalmente dos o tres intervenciones). Además, los nuevos tratamientos que se desarrollan son investigados mediante la realización de ensayos clínicos controlados que comparan generalmente el nuevo tratamiento solo con algunas de las alternativas existentes y no con todas ellas. Se elige principalmente el patrón oro o gold standard, o el competidor más importante del mercado, lo que aumenta la falta de comparaciones directas entre las alternativas de intervención a medida que avanzan los años. Esto es debido a que no hay un incentivo para que las empresas desarrolladoras de nuevas tecnologías inviertan recursos en comparar todas las alternativas existentes, por lo tanto, las empresas solo investigan las alternativas que a su juicio les reportan mayores beneficios económicos. Esta brecha de la falta de comparaciones no solo está lejos de cerrarse sino que se va a ir agravando mucho más en el futuro.

Desde el punto de vista de las técnicas utilizadas en la medicina basada en la evidencia, cuando no existe un ensayo clínico controlado que compare las alternativas directamente, se utiliza un estudio con menor 
nivel de evidencia. La calidad de la evidencia disminuye a estudios observacionales descriptivos o incluso a series de casos y opiniones de expertos, buscando la mejor evidencia que permita tomar una decisión, ya que no existe la comparación directa ideal. Pero, ¿es esto lo más correcto? Cuando no existe una comparación directa entre dos tratamientos se genera la siguiente disyuntiva: ¿qué es mejor, tomar la decisión con una serie de casos o una opinión de expertos, o estimar matemáticamente cual sería la comparación indirecta, mediante la información directa que sí existe, entre los diferentes estudios? En estos casos, la realización de una comparación indirecta entre tratamientos mediante un metaanálisis en red permite tener información importante para la toma de decisiones, que complementa la información disponible ${ }^{1,5}$.

La toma de decisiones en salud se basa por lo menos en los resultados y en los costos de las intervenciones involucradas. Por lo tanto involucran modelamientos matemáticos de alternativas múltiples, propios de los análisis económicos. Los árboles de decisión y las cadenas de Markov son los más utilizados en estudios de costo-utilidad o costo-efectividad. En estas investigaciones, los metaanálisis en red son muy útiles, ya que proveen las probabilidades que son incorporadas en los modelos para realizar estos análisis múltiples ${ }^{1}$.

Por ejemplo, realizar un estudio para identificar qué medicamento hipotensor ocular es más efectivo en la reducción de la tensión ocular en el tratamiento de pacientes con glaucoma crónico de ángulo abierto implica la comparación de 9 medicamentos diferentes ${ }^{6}$. Al realizar una revisión sistemática, seleccionamos ensayos clínicos controlados, y la limitante es la comparación de tres o menos hipotensores simultáneamente. Si se realiza un metaanálisis, las comparaciones solo se pueden hacer con 2 tratamientos en cada análisis: el riesgo relativo (RR) analiza 2 alternativas simultáneamente, por lo tanto no podemos obtener las relaciones entre los 9 tratamientos disponibles. Si además se desea realizar un estudio del análisis económico incorporando los costos para conocer qué tratamiento tiene una mejor relación costoefectiva, se deben incorporar las probabilidades en un modelo de árbol de decisión, lo que nos obliga a estimar las relaciones entre estos 9 tratamientos mediante un metaanálisis en red. Esto permite correr el modelo y complementar la información preexistente para la toma de decisiones.

El concepto de metaanálisis en red fue propuesto por Lumley en el año $2002^{2}$. Proporcionó un marco estadístico unificado para la comparación de múltiples tratamientos. Hasta el año 2005, la cantidad de artículos de metaanálisis en red era mínima. Desde el año 2006 se ha producido un aumento exponencial de este tipo de estudios: cada año se realizan cientos de estudios de este tipo y son cada vez más utilizados como fuentes de estimación. Ante la falta de análisis directos en algunas áreas médicas, las agencias internacionales de evaluación (National Institute for Health and Care Excellence [NICE] en el Reino Unido, Canadian Agency for Drugs and Technologies in Health [CADTH] en Canadá, Pharmaceutical Benefits Advisory Committee [PBAC] en Australia, Haute Autorité de Santé [HAS] en Francia, Institute for Quality and Efficiency in Health Care [IQWIG] en Alemania, o incluso International Society for Pharmacoeconomics and Outcomes Research [ISPOR] $)^{1}$ consideran que las comparaciones indirectas son una fuente de información en la toma de decisiones en salud, para lo cual cada agencia ha publicado sus propias normas al respecto. Existe además una ampliación de la declaración Preferred Reporting Items for Systematic Reviews and Meta-Analysis (PRISMA), la PRISMA-Network Meta-analysis (NMA), que permite orientar el análisis de los estudios de forma más estructurada 7 . Por lo tanto se vuelve cada vez más necesario conocer este tipo de estudios y saber cómo interpretar sus resultados.

El objetivo de esta revisión narrativa es describir los conceptos fundamentales de los metaanálisis en red, utilidad, conformación de la red, consideraciones metodológicas y sus principales limitaciones.

\section{¿Qué es un metaanálisis en red?}

El metaanálisis en red (network meta-analysis) ${ }^{1-5}$, es una técnica estadística que combina estudios experimentales con similares características y que no han sido comparados directamente mediante la aplicación de una red de análisis. Este análisis integrado y unificado incorpora toda la información de comparaciones directas e indirectas sobre los diferentes tratamientos y permite así calcular los efectos relativos de todos los tratamientos incluidos en la red. Se estiman utilizando tanto los análisis directos como los indirectos (Fig. 1).

Se refiere a "comparación directa» cuando 2 tratamientos se han evaluado ( $A$ vs. B y A vs. C) el uno contra el otro y a "comparación indirecta» si dos tratamientos nunca se han comparado uno contra el otro directamente (B vs. $C$ ), pero estos dos tratamientos se han comparado contra un comparador común (A, placebo): se puede realizar así una comparación indirecta entre los tratamientos utilizando los efectos relativos de 

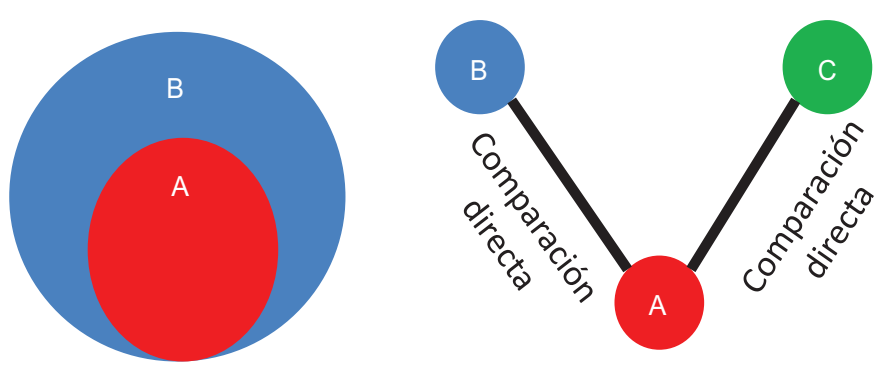

Comparador común
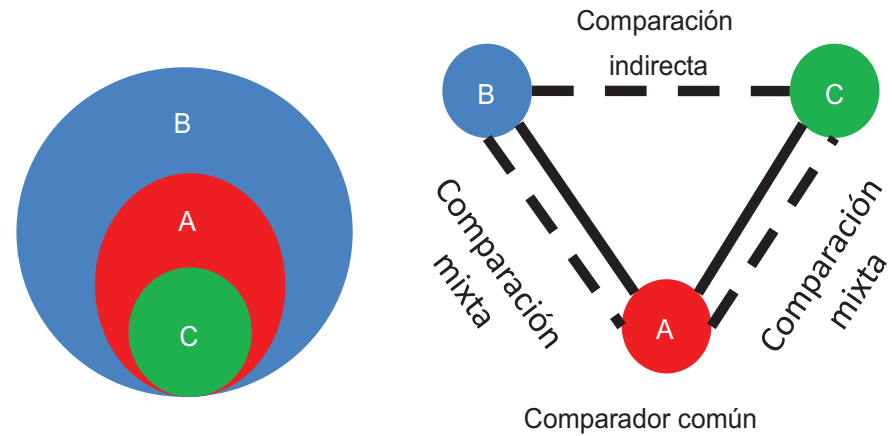

Figura 1. Comparaciones directas, indirectas y mixtas.

los 2 tratamientos frente al comparador en común. En la figura 1, cada nodo (círculo) refleja una intervención o comparador (placebo, tratamiento, etc.) y la línea que conecta a dos nodos refleja uno o más ensayos clínicos. En algunos diagramas se cambia el tamaño del nodo para representar la mayor o menor importancia del estudio y también se puede hacer la línea más gruesa o delgada para representar visualmente si es un estudio o son muchos ${ }^{8-10}$. Aunque a menudo se argumenta que las comparaciones indirectas solo son necesarias cuando las comparaciones directas no existen, es importante tener en cuenta que los resultados de pruebas indirectas en combinación con las pruebas directas pueden reforzar la evaluación entre los tratamientos ${ }^{11,12}$. Las estimaciones indirectas ofrecen una estimación adicional del efecto del tratamiento que complementa a la directa. En algunos casos la estimación directa puede provenir de la comparación de ensayos clínicos controlados de tamaño pequeño o con algunos problemas metodológicos, mientras que la comparación indirecta puede provenir de la comparación de ensayos clínicos controlados de muy buena calidad y ser esta más consistente y eficiente que la estimación directa.

\section{Conformación de la red}

Una red está compuesta por al menos tres nodos conectados por líneas. Existen dos tipos diferentes de redes que muestran cómo se comparan los tratamientos: de bucles abiertos y de bucles cerrados (Fig. 2) $)^{1,2,11,12}$. Se relaciona con la cantidad de estudios que existen con comparaciones directas entre las diferentes alternativas. Cuando hay al menos un estudio que compara una alternativa con otra y permite que todas las alternativas queden conectadas unas con otras en el diagrama de la red, se habla de «bucles cerrados" (Fig. 2 C). Pero si las comparaciones directas no existen, se habla de bucles abiertos (Fig. 2 A y B).

En las redes de bucles abiertos se compara el efecto relativo entre $B$ y $C$ (Fig. 2 A) mediante una comparación indirecta anclada a A (en la bibliografía también se habla de "ajustado"). Es fundamental en la comparación que no se rompa la aleatorización ${ }^{8,13,14}$, por lo que no se deben utilizar solo los datos de los grupos de tratamiento y generar de ahí las comparaciones omitiendo el grupo control o anclado. Cuando se realiza este tipo de comparación se denomina "comparación indirecta simplista» y genera sesgos: no debería 

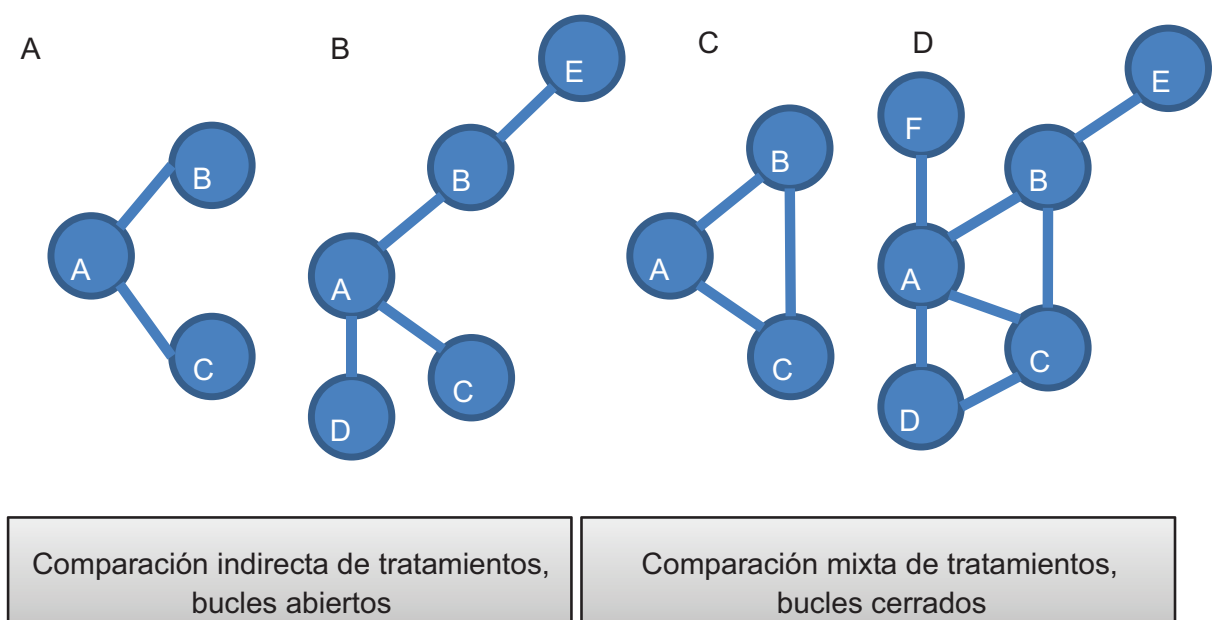

Comparación mixta de tratamientos, bucles cerrados
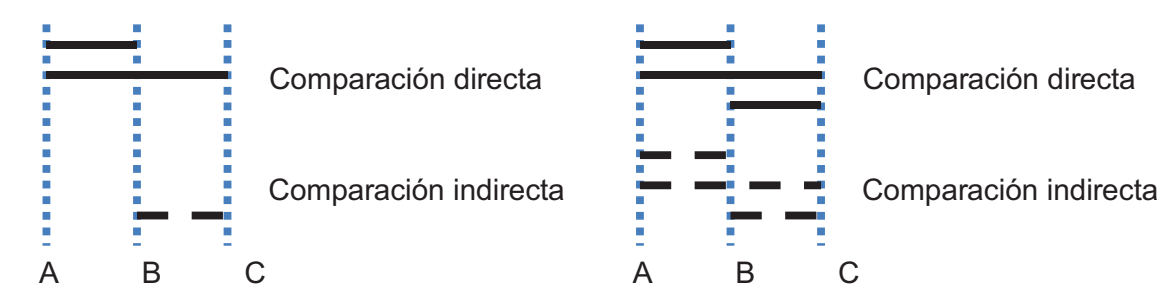

Figura 2. Tipos de redes de metaanálisis. En el sector izquierdo se aprecian ejemplos de red de bucles abiertos; en el sector derecho, de bucles cerrados, y en la zona inferior se aprecian las diferencias que existen en la cantidad de comparaciones entre estudios para las redes A y $\mathrm{C}$.

realizarse. Para conservar la aleatorización se deben comparar los efectos relativos, se compara la razón de probabilidad de $B$ vs. A con la razón de probabilidad de $C$ vs. $A^{1,14}$. Cuando hay múltiples tratamientos en torno a un grupo control (Fig. 2 B), se habla también de «red en forma de estrella». En este caso, no todos los estudios clínicos tienen un mismo comparador o anclaje ( $B$ vs. $E$ ), pero todas las intervenciones deben permanecer conectadas unas con otras.

Matemáticamente, en los bucles cerrados, para cada comparación de 2 alternativas existen 2 fuentes de información: las estimaciones del efecto obtenidas directamente e indirectamente (Fig. 2 C). En estos casos, la red se denomina "comparación mixta de tratamientos" (mixed treatment comparison). En la práctica, las redes muy grandes, con muchas alternativas, pueden en muchos casos ser una mezcla con zonas de ambos bucles (Fig. 2 D).

Cuando hay un bucle abierto, el riesgo de sesgo de la estimación es mayor que cuando no lo hay. Cuando ambas pruebas, directa e indirecta, están disponibles, las dos fuentes de información pueden ser combinadas como una media ponderada. Este tipo de comparación se puede extender a k comparaciones, para facilitar la inferencia simultánea sobre todos los tratamientos disponibles y aportar mayor información en la selección de las opciones de tratamiento o de las intervenciones ${ }^{11,12,14}$. Incluso cuando los resultados de las comparaciones directas son concluyentes, al combinarlas con los resultados de las estimaciones indirectas en una comparación de tratamiento mixto puede obtenerse una estimación más precisa para las intervenciones y se amplia la población a partir de la cual se infieren los resultados. Todos los bucles son utilizados para realizar el análisis, pero cuanto más lejos están los bucles de la estimación que se está calculando (o sea, que la estimación se deriva de un análisis indirecto más lejano dentro de la red) menor peso a nivel de la estimación indirecta calculada tienen estos bucles ${ }^{11,12}$. Finalmente, en los cálculos obtenidos debe haber consistencia entre las estimaciones directas e indirectas con bucles cerrados, o sea, el valor estimado de forma directa debe ser similar al valor estimado de forma indirecta. Si esto no ocurre, se habla de "cálculos inconsistentes» $y$, por ende, las estimaciones deben ser interpretadas con mucha cautela mientras no se 
identifique la causa de la diferencia. En estos casos debe procederse de manera similar que en un metaanálisis típico: si las fuentes incluidas en el análisis cumplen con los criterios adecuados de calidad de un ensayo clínico controlado, las estimaciones inconsistentes dan cuenta de la incertidumbre de la estimación o de la heterogeneidad real de las poblaciones de estudio en relación con el efecto del tratamiento. El desafío no es la elección entre la estimación directa o indirecta, sino más bien la adecuada caracterización de esa incertidumbre para facilitar el proceso de toma de decisiones.

\section{Limitaciones}

La comparación indirecta obtenida no es directamente aleatorizada, como en el caso de la comparación directa. Es observacional, por lo que está sujeta a más sesgos potenciales que la comparación directa ${ }^{1,11,12}$. En los ensayos clínicos controlados bien realizados y aleatorizados se obtienen estimadores con sesgos mínimos del efecto. Teóricamente, al ser utilizados estos para generar una estimación indirecta, la estimación calculada resulta muy poco sesgada también. No obstante, en la práctica, frecuentemente, los ensayos clínicos controlados no son idénticos metodológicamente y la comparación indirecta depende de la asunción de similitud o transitividad de los estudios $^{14}$ : si el tratamiento $B$ es mejor que $A$ y $A$ es mejor que $C$ se asume que el tratamiento $B$ es mejor que $C$ (Fig. 1). Pero existen estudios en los que se discute el incumplimiento de la transitividad, ya que no siempre se da $^{8}$. Esta asunción depende de lo parecidas que sean las metodologías empleadas, de la mezcla de estudios muy antiguos con otros nuevos, de los grupos de pacientes, etc. En estos aspectos, los metaanálisis en red no son muy diferentes a los metaanálisis normales: ambos requieren de la búsqueda sistemática y exhaustiva de todos los artículos potencialmente relevantes, la selección de los artículos mediante criterios explícitos y reproducibles, que serán incluidos, la descripción del diseño y la ejecución similar de los estudios originales, la síntesis de los datos obtenidos y resultados (outcome) medidos de forma similar. Por lo tanto, la interpretación de los resultados se realiza teniendo en cuenta el proceso de investigación y la red, más que en la medida matemática de resumen obtenida. Todo este proceso tiene el riesgo potencial de producir sesgos en el estudio y, así, ofrecer unas conclusiones no válidas ${ }^{1,2,11,12,14}$. Song, et al. ${ }^{13}$ estimó la inconsistencia de los metaanálisis en red entre la comparación directa e indirecta medida mediante la odds ratio (OR) calculada. Este fue estadísticamente significativo en el 14\% de los casos (IC 95\%: 9-22). Veroniki, et al. $2013^{15}$ encontró inconsistencia en los resultados del 2 al $9 \%$ de los casos, dependiendo de la medida del efecto y de la estimación de la heterogeneidad. En ambos estudios se estima que la consistencia está en torno a un $85 \%$ de los $\operatorname{casos}^{16}$, o sea, que las estimaciones obtenidas de forma indirecta y las obtenidas de forma directa coinciden en un $85 \%$ de las investigaciones estudiadas.

Aunque los metaanálisis en red se recomiendan generalmente solo para ensayos clínicos controlados con asignación al azar, la metodología de los metaanálisis en red podría aplicarse a situaciones en las que los datos se obtienen a partir de otros tipos de investigación.

\section{Consideraciones metodológicas}

Se utilizan varios métodos estadísticos para realizar los cálculos en los metaanálisis en red ${ }^{11,12}$. Se pueden realizar con modelos de enfoques frecuentistas de efectos fijos o aleatorios, modelos de metarregresión o modelos bayesianos, entre otros. Algunos enfoques proporcionan una mayor flexibilidad que otros para ajustar por covariables y análisis de intervenciones múltiples. La mayoría de los metaanálisis en red utilizan un enfoque estadístico único. El más frecuente es el modelo bayesiano. La comparación del rendimiento de los diferentes métodos no se ha estudiado en detalle. En estudios metodológicos futuros se puede evaluar la utilidad y robustez de los diversos métodos estadísticos y determinar las circunstancias en las que un método específico es más eficiente y apropiado que otro.

\section{Software estadísticos}

Los programas más utilizados para el análisis de los diferentes modelos y su representación gráfica son: WinBUGS, OpenbBUGS, JAGS (Just Another Gibbs Sampler), SAS ${ }^{\circledR}$ (Statistical Analysis System), STATA ${ }^{\circledR}$ (con comando MV_META) ${ }^{9}, \mathrm{R}$ (con paquetes metaphor, GeMTC, pcnetmeta y netmeta $)^{17}$ y Excel ${ }^{18}$. Permiten su programación para tales fines mediante distintos comandos de análisis disponibles y representaciones gráficas. El manejo de estos programas requieren de vastos conocimientos estadísticos para crear y correr los modelos de análisis en redes. 


\section{Conclusiones}

El metaanálisis en red es un tipo de investigación muy útil para comparar múltiples tratamientos o frente a la falta de comparaciones directas entre tratamientos. Su uso es complementario a las evaluaciones directas cuando estas existen, no las sustituye en ningún caso. Si las comparaciones indirectas provienen de bucles cerrados, proveen mucha más información y menos sesgada que de bucles abiertos. Los metaanálisis en red pueden ser muy informativos y útiles, pero, dadas las asunciones utilizadas, la confianza en la estimación global no siempre es alta. Aún está por definir cuáles son los métodos estadísticos adecuados para cada tipo de análisis y el desarrollo de programas computacionales que permitan los análisis de forma automática y segura. En consecuencia, actualmente es más importante el análisis del proceso y de la red de intervenciones que la obtención de una medida ponderada final.

\section{Responsabilidades éticas}

Protección de personas y animales. Los autores declaran que para esta investigación no se han realizado experimentos en seres humanos ni en animales.

Confidencialidad de los datos. Los autores declaran que en este artículo no aparecen datos de pacientes.

Derecho a la privacidad y consentimiento informado. Los autores declaran que en este artículo no aparecen datos de pacientes.

\section{Financiamiento}

No hay financiación de otras instituciones.

\section{Conflicto de intereses}

No hay conflicto de intereses.

\section{Bibliografía}

1. Jansen JP, Trikalinos T, Cappelleri JC, Daw J, Andes S, Eldessouki R, et al. Indirect Treatment Comparison/Network Meta-Analysis Study Questionnaire to Assess Relevance and Credibility to Inform Health Care Decision Making: An ISPOR-AMCP-NPC Good Practice Task Force Report. Value in Health. 2014;17(2):157-73.

2. Lumley T. Network meta-analysis for indirect treatment comparisons. Stat Med. 2002;21(16):2313-24.

3. Li T, Puhan MA, Vedula SS, Singh S, Dickersin K. Network meta-analysis-highly attractive but more methodological research is needed. BMC Med. 2011;9:1-5.

4. Van Valkenhoef G, Tervonen T, Zhao J, De Brock B, Hillege HL, Postmus D. Multicriteria benefit-risk assessment using network meta-analysis. J Clin Epidemiol. 2012;65(4):394-403.

5. Haynes RB. Of studies, syntheses, synopses, summaries, and systems: the " $5 S$ " evolution of information services for evidence-based healthcare decisions. Evid Based Med. 2006;11(6):162-4.

6. Van der Valk $R$, Webers CA, Lumley $T$, Hendrikse $F$, Prins $M H$ Schouten JS. Network meta-analysis combined direct and indirect comparisons between glaucoma drugs to rank effectiveness in lowering intraocular pressure. J Clin Epidemiol. 2009;62(12):1279-83.

7. Hutton B, Salanti G, Caldwell DM, Chaimani A, Schmid CH, Cameron C, et al. The PRISMA extension statement for reporting of systematic reviews incorporating network meta-analyses of health care interventions: checklist and explanations. Ann Intern Med. 2015;162(11):777-84

8. Chung $\mathrm{H}$, Lumley T. Graphical exploration of network meta-analysis data: the use of multidimensional scaling. Clin Trials. 2008;5(4):301-7.

9. Chaimani A, Higgins JP, Mavridis D, Spyridonos P, Salanti G. Graphical tools for network meta-analysis in STATA. PLoS One. 2013; 8:e76654.

10. Zhang J, Xie J, Hou W, Tu X, Xu J, Song F, et al. Mapping the knowledge structure of research on patient adherence: knowledge domain visualization based co-word analysis and social network analysis. PLoS One. 2012;7(4):e34497.

11. Jones B, Roger J, Lane PW, Lawton A, Fletcher C, Cappelleri JC, et al. Statistical approaches for conducting network meta-analysis in drug development. Pharm Stat. 2011;10(6):523-31.

12. Song F, Clark A, Bachmann MO, Maas J. Simulation evaluation of statistical properties of methods for indirect and mixed treatment comparisons. BMC Med Res Methodol. 2012;12:138.

13. Song F, Xiong T, Parekh-Bhurke S, Loke YK, Sutton AJ, Eastwood AJ, et al. Inconsistency between direct and indirect comparisons of competing interventions: meta-epidemiological study. BMJ. 2011; 343:d4909.

14. Catalá-López F, Hutton B, Moher D. La propiedad transitiva en los ensayos clínicos controlados: si $B$ es mejor que $A$ y $C$ es mejor que $B, ~ ¿ C$ será mejor que A? Rev Esp Cardiol. 2014;67(8):597-602.

15. Veroniki AA, Vasiliadis HS, Higgins JP, Salanti G. Evaluation of inconsistency in networks of interventions. Int J Epidemiol. 2013;42(1):332-45.

16. Song F, Loke YK, Walsh T, Glenny AM, Eastwood AJ, Altman DG. Methodological problems in the use of indirect comparisons for evaluating healthcare interventions: survey of published systematic reviews. BMJ. 2009;338(7700):932-5

17. Neupane B, Richer D, Bonner AJ, Kibret T, Beyene J. Network Meta-Analysis Using R: A Review of Currently Available Automated Packages. PLoS One. 2015;10(4):e0123364.

18. Tobías A, Catalá-López F, Roqué M. Desarrollo de una hoja Excel para metaanálisis de comparaciones indirectas y mixtas. Rev Esp de Salud Pública. 2014;88(1):5-15. 